\title{
Editorial: (Pushing) the Limits of Neuroplasticity Induced by Adult Language Acquisition
}

\author{
Jurriaan Witteman ${ }^{1,2,3 *}$, Yiya Chen ${ }^{1,2,3}$, Leticia Pablos-Robles ${ }^{1,2,3}$, \\ Maria Carmen Parafita Couto ${ }^{1,2,3}$, Patrick C. M. Wong ${ }^{4}$ and Niels O. Schiller ${ }^{1,2,3}$ \\ ${ }^{1}$ Department of Linguistics, Leiden University, Leiden, Netherlands, ${ }^{2}$ Leiden University Centre for Linguistics, Leiden, \\ Netherlands, ${ }^{3}$ Leiden Institute for Brain and Cognition, Leiden University, Leiden, Netherlands, ${ }^{4}$ Department of Linguistics \\ and Modern Languages, Brain and Mind Institute, The Chinese University of Hong Kong, Hong Kong, China
}

Keywords: brain, cognition, language, bilingualism, neuroplasicity

\section{Editorial on the Research Topic}

\section{(Pushing) the Limits of Neuroplasticity Induced by Adult Language Acquisition}

Many individuals attempt to learn a second (L2) or even third language (L3) at some point in their life. Since language exposure is one of the most intense cognitive training regimes one can encounter, it is not surprising that previous research has shown that multilingualism can induce profound neural changes or "neuroplasticity" (Costa and Sebastián-Gallés, 2014). Despite the general consensus that learning a new language in adulthood can change the brain, what remains unclear is the scope of such neuroplasticity. In other words, what limits vs. promotes neurocognitive change as a result of second language acquisition in adulthood?

On the one hand, there are factors that may limit such change of the neurocognitive system due to L2 (or L3) acquisition. For instance, models of adult L2 learning assume that acquisition of the mother tongue (L1) has sculpted neural circuits to discriminate between L1 linguistic elements which in turn limits the ability to distinguish between L2 elements (e.g., van Leussen and Escudero, 2015). On the other hand, there might be factors that enhance L2 induced neurocognitive change, such as language aptitude (Hu et al., 2013; Chai et al., 2016) and the intensity (Tremblay et al., 1997; Thomson and Derwing, 2015) and quality (Zhang et al., 2009; Ylinen et al., 2010; Morgan-Short et al., 2012; Grimaldi et al., 2014) of the L2 acquisition regime. Hence, much is yet to be investigated about the factors that limit vs. promote adult language learning induced neuroplasticity as well as the mediating underlying neurocognitive mechanisms. The present research topic therefore aimed to identify some of the factors that limit or promote adult L2 learning induced neurocognitive plasticity and the underlying neurocognitive mechanisms.

What factors then, might limit neurocognitive change due to adult L2 acquisition? The two reviews presented in the current research topic (Birdsong; Antoniou and Wright) both suggest that having reached adult age itself might be a limiting factor because adult age represents a period of relatively (as compared to childhood) low susceptibility to L2 exposure, limiting the degree to which L2 proficiency can be gained. Additionally, the mismatch of L1-L2 typology was suggested to limit L2 acquisition, with a relatively large mismatch delaying successful L2 acquisition (Antoniou and Wright). Indeed, a cross-linguistic priming study with concurrent ERP recordings presented in the current research topic showed that already early in the L2 acquisition process there is interaction between L1 and L2 at the semantic level (Meade et al.). Another study (Yang et al.), showed that a large typological difference between L1 and an L3 makes switching between languages in bilinguals more difficult with different underlying cognitive control networks being engaged in switching between balanced vs. unbalanced languages. 
Acquisition of L2 (and underlying neurocognitive change) may additionally vary between domains of the language or even within a domain. Indeed, a study presented in the current research topic showed that while learning an artificial language, words that correspond to relatively concrete concepts are more easily integrated into existing semantic networks than words that refer to relatively abstract concepts (Ding et al.).

Finally, as outlined above (adult) age may itself limit neuroplastic change due to L2 learning. Indeed, a structural white matter imaging study presented in the current research topic suggests that white matter bundles critical for obtaining syntactic abilities are still developing in adolescence but may have reached maturation in adults (Yamamoto and Sakai), perhaps limiting acquisition of L2 syntax at adult age. On the other hand, the limiting effect of age of acquisition on L2 induced structural neuroplasticity may in itself be limited, as shown by a study presented in the current research topic, demonstrating white matter differences purportedly due to L2 learning between mono- and bilinguals, despite of L2 learners having reached adulthood (Rossi et al.).

Having discussed the factors that may limit neuroplasticity due to adult L2 acquisition, what factors may promote it? One of the reviews of the present research topic (Birdsong) mentions some possible factors such as: high working memory capacity, motivation to learn and meta-linguistic awareness (which could be promoted by having successfully acquired a previous nonnative language). Indeed, a study in the present research topic examining predictors of L2 acquisition success found evidence that high working memory capacity predicts L2 acquisition success (Blumenfeld et al.). Furthermore, the general ability

\section{REFERENCES}

Chai, X. J., Berken, J. A., Barbeau, E. B., Soles, J., Callahan, M., Chen, J.K., et al. (2016). Intrinsic functional connectivity in the adult brain and success in second-language learning. J. Neurosci. 36, 755-761. doi: 10.1523/JNEUROSCI.2234-15.2016

Costa, A., and Sebastián-Gallés, N. (2014). How does the bilingual experience sculpt the brain? Nat. Rev. Neurosci. 15, 336-345. doi: 10.1038/nrn3709

Grimaldi, M., Sisinni, B., Gili Fivela, B., Invitto, S., Resta, D., Alku, P., et al. (2014). Assimilation of L2 vowels to L1 phonemes governs L2 learning in adulthood: a behavioral and ERP study. Front. Hum. Neurosci. 8:279. doi: 10.3389/fnhum.2014.00279

Hu, X., Ackermann, H., Martin, J. A., Erb, M., Winkler, S., and Reiterer, S. M. (2013). Language aptitude for pronunciation in advanced second language (L2) Learners: Behavioural predictors and neural substrates. Brain Language 127, 366-376. doi: 10.1016/j.bandl.2012.11.006

Morgan-Short, K., Steinhauer, K., Sanz, C., and Ullman, M. T. (2012). Explicit and implicit second language training differentially affect the achievement of native-like brain activation patterns. J. Cogn. Neurosci. 24, 933-947. doi: 10.1162/jocn_a_00119

Thomson, R. I., and Derwing, T. M. (2015). The effectiveness of L2 pronunciation instruction: a narrative review. Appl. Linguist. 36, 326-344. doi: 10.1093/applin/amu076

Tremblay, K., Kraus, N., Carrell, T. D., and McGee, T. (1997). Central auditory system plasticity: generalization to novel stimuli following listening training. J. Acoust. Soc. Am. 102, 3762-3773. to learn or "language aptitude" may enhance neurocognitive change induced by L2 or L3 learning. Indeed, a study presented in our research topic investigating the morphology of Heschl's gyrus (HG), the primary auditory cortex, suggests that the number of complete duplications of HG in the right hemisphere might be a structural correlate of language aptitude, that may enhance L2 acquisition success (Turker et al.). Finally, in an interesting study examining the effects of L2 acquisition on L1 processing presented in the current research topic (Kasparian and Steinhauer), very extended exposure to L2 and resulting high L2 proficiency emerged as an important factor in determining (abnormal) morphosyntactic L1 processing, suggesting that the intensity of L2 exposure is a critical determinant of neuroplastic change in the underlying neurocognitive architecture of the language processing system.

In sum, the studies presented in the current research topic suggest that neuroplastic change due to acquisition of another language (L2, L3, etc.) seems to be limited by adult age, typological mismatch between the already acquired and to be acquired languages, and limited exposure to the to be acquired language. On the other hand, high working memory capacity, high "language aptitude," and a high level of exposure to the to be acquired language seem to promote neuroplastic change. Together, we have aimed with the studies presented in the current research topic to provide a fresh look at the scope of neuroplastic change due to adult second language acquisition.

\section{AUTHOR CONTRIBUTIONS}

Conceived the idea: JW and NS; Wrote the Research Topic proposal: JW, YC, LP-R, MP, PW, and NS; Edited articles: JW, YC, LP-R, MP, PW, and NS; Wrote the editorial: JW.

van Leussen, J.-W., and Escudero, P. (2015). Learning to perceive and recognize a second language: the L2LP model revised. Front. Psychol. 6:1000. doi: 10.3389/fpsyg.2015.01000

Ylinen, S., Uther, M., Latvala, A., Vepsäläinen, S., Iverson, P., Akahane-Yamada, R., et al. (2010). Training the brain to weight speech cues differently: a study of finnish second-language users of english. J. Cogn. Neurosci. 22, 1319-1332. doi: 10.1162/jocn.2009.21272

Zhang, Y., Kuhl, P. K., Imada, T., Iverson, P., Pruitt, J., Stevens, E. B., et al. Nemoto, I. (2009). Neural signatures of phonetic learning in adulthood: A magnetoencephalography study. Neuroimage 46, 226-240. doi: 10.1016/j.neuroimage.2009.01.028

Conflict of Interest Statement: PW is co-owner of a tech startup company in Hong Kong that is related to this research topic.

The remaining authors declare that the research was conducted in the absence of any commercial or financial relationships that could be construed as a potential conflict of interest.

Copyright (c) 2018 Witteman, Chen, Pablos-Robles, Parafita Couto, Wong and Schiller. This is an open-access article distributed under the terms of the Creative Commons Attribution License (CC BY). The use, distribution or reproduction in other forums is permitted, provided the original author(s) and the copyright owner(s) are credited and that the original publication in this journal is cited, in accordance with accepted academic practice. No use, distribution or reproduction is permitted which does not comply with these terms. 